\title{
Enhancing Quality of Life for Children with Intellectual and Developmental Disabilities
}

Erin E. SNAPP ${ }^{* \text { (D) }}$, Leah R. KETCHESON ${ }^{\text {(D) }}$, Jeffrey J. MARTIN ${ }^{\text {(D) }}$, Erin E. CENTEIO ${ }^{\text {(D) }}$

\footnotetext{
${ }^{1}$ Wayne State UniversityDetroit, Michigan, USA

${ }^{2}$ University of Hawai'i Mānoa, Honolulu, Hawai'i, USA

*Corresponding author: Gd3103@wayne.edu
}

\begin{abstract}
Background: Quality of life (QOL) is a subjective appraisal of life conditions. Disabilities often decrease QOL for individuals, due to potential physical, cognitive, and social limitations. Children with intellectual and developmental disabilities (IDD) have delayed fundamental motor skills (FMS) and less physical activity compared to neurotypical peers resulting in lower QOL. The purpose of this study is to provide evidence that FMS interventions improve QOL for children with IDD. Objectives/Hypothesis: In the current study we evaluated the impact of three FMS interventions over 18 months on QOL for 10 children (age range from 4 to $14, M=8.88, S D=2.93$ ) with IDD. Methods: The FMS intervention took place over three separate 10 week sessions. Using the Pediatric Quality of Life Scale 4.0 we measured physical, social, emotional, and school functioning QOL. Visual analysis was used to evaluate the group means of each subscale across six time points, revealing a trend toward improved QOL for all 4 QOL subscales. Results: Emotional and social function had the largest improvements. This study addresses the need for longitudinal research on how FMS interventions can enhance QOL for children with IDD. Conclusions: Community based programs focused on FMS can help improved QOL for children with IDD. This research helps to better understand how FMS interventions can improve several areas of life.
\end{abstract}

Keywords

Fundamental Motor Skills; Visual Analysis; Longitudinal Research; Intellectual and Developmental Disabilities

\section{INTRODUCTION}

The World Health Organization (WHO, 1948) has defined health "as a state of complete physical, mental and social well-being." Historically many researchers have focused solely on the physical aspect in their definition of health. However, the WHO (1948) definition began the inclusion of psychological and social concepts into health resulting in a more holistic approach. The idea of health as a multidimensional concept was instrumental in the development of quality of life (QOL) as a measurable construct. Broadly, QOL can be defined as the subjective and objective appraisal of life conditions for each individual (Felce \& Perry, 1995). This appraisal can be measured for multiple domains of life including physical functioning, emotional functioning, social relationships, and more (Guyatt, Feeny \& Patrick, 1993). Given the different domains, one can expect that personal perceptions of many factors such as physical activity, depression, anxiety, friendships, and self-esteem can potentially influence QOL (Muldoon, Barger, Flory \& Manuck).

Quality of life can also be greatly impacted by disability or illness. Over time, health-related QOL became the preferred term for assessments measuring the impact of disease or disability and particularly how it relates to daily functioning (Wood-Dauphinee, 1999). Individuals with disabilities and individuals diagnosed with a disease or illness may experience difficulty with common activities of daily life compared to individuals without disabilities. These impairment- 
related challenges can potentially reduce health related QOL. Health related QOL works to encompass the impact on important aspects of daily life while including aspects that might not generally be considered health (Guyatt, Feeny \& Patrick, 1993).

When assessing QOL, researchers have been specific and varied in their definition of QOL. As a result, this has led to hundreds of QOL definitions and a variety of different measures. With the diversity of definitions, comparison across studies using different measures has become almost impossible (Wallander, Schmitt \& Koot, 2001; Wood-Dauphinee, 1999). Further, this leads to little opportunity for generalization of results about QOL across studies. Despite these differences, a consensus has emerged that multiple components of QOL are important for a comprehensive measure and subsequent understanding of QOL (Wallander et al., 2001).

Some individuals such as those with intellectual disabilities or young children may not have the ability to effectively complete QOL subjective assessments. Therefore, oftentimes, measures of QOL are completed by proxy, such as a parent or primary caregiver (Pickard \& Knight, 2005). Upton and colleagues (2008) conducted a review of parent-child agreement across health related QOL measures. They found that there were some differences in scores between the parent and child, but the differences don't appear to be clinically significant between respondents (Upton, Lawford \& Eiser, 2008). In general, scores for more concrete areas of functioning, such as physical functioning, show better agreement between the participant and their proxy, while proxies often rated more subjective aspects, such as social or emotional functioning, worse than the participant (Pickard \& Knight, 2005). Researchers have speculated about the potential reasons for this, including proxies projecting their own negative feelings or the participants decreased awareness of their own deficits (Potvin, Snider, Prelock, Wood-Dauphinee \& Kehayia, 2015). Nonetheless, parent or caregiver proxy is a commonly used method for assessment of QOL for individuals unable to complete the measures on their own.

Many researchers have examined QOL for individuals with disabilities (Martin, 2017). However, research on QOL for children with intellectual and developmental disabilities (IDD) is limited. Intellectual and developmental disabilities are characterized by deficits in intellectual functioning and/or impairment in adaptive behavior identified during the developmental period (American Psychiatric Association, 2013). The limited literature in this area is often focused on the QOL of parents and families of children with IDD (Brown, Schalock, \& Brown, 2009; Peer $\&$ Hillman, 2014). Far fewer research efforts have focused on the outcomes of the child.

It is well established that physical activity (PA) participation is beneficial for health outcomes, such as maintaining a healthy weight status and body composition, preventing heart disease and diabetes, and improving physical functioning (Penedo \& Dahn, 2005). However, it is also valuable for improving psychological wellbeing (Warburton, Nicol \& Bredin, 2006), academic achievement (Strong et al., 2005), and enhancing overall QOL (Penedo \& Dahn, 2005). Despite the value of PA, many able-bodied children do not meet PA guidelines. An even smaller percentage of children with IDD meet the PA recommendations compared to their neurotypical peers (Esposito, MacDonald, Hornyak, \& Ulrich, 2012; Tyler, MacDonald, \& Menear, 2014). The lower rates of PA for children with IDD often results from increased barriers to PA participation. These additional barriers can stem from lack of financial resources, limited social support, and poor fundamental motor skill development (Gregor et al., 2018; Memari et al., 2015). By intervening on fundamental motor skills (FMS) at an early age, school aged children are more likely to engage in regular PA across childhood (Robinson et al., 2015). However, while evidence-based guidelines for early FMS interventions exist, the opportunity to engage in such programming to enhance QOL for children with IDD is virtually non-existent. The current study seeks to address this research gap.

\section{Purpose}

To summarize, the purpose of the current study was to evaluate the impact of longitudinal participation in a free community-based FMS intervention on QOL for children with IDD. We hypothesized that all areas (physical, emotional, social and school functioning) of QOL would increase during a series of 3 interventions spread over 1.5 years. More specifically, we hypothesized that all 4 dimensions of QOL would increase between each pre- and post-intervention data 
collection session. However, given the physical and social nature of the intervention we expected to see larger gains in physical and social QOL compared to emotional and school functioning. Additionally, we hypothesized that QOL would stay the same between the end of each intervention and the beginning of the next intervention given that the intervention was removed.

\section{METHODS}

\section{Participants}

Participants were recruited through local schools and agencies for those with special needs. The sample consisted of ten children diagnosed with various IDD. The majority of the sample reported a primary diagnosis of Autism Spectrum Disorder (ASD) $(n=8)$, with some reporting comorbid speech deficits $(\mathrm{n}=2)$, other primary diagnoses included Down syndrome and Fragile X syndrome. Age of participants at the initial data collection ranged between 4 and 14 years of age $(M=8.88, S D=2.93)$.

\section{Measure}

\section{Pediatric Quality of Life Inventory 4.0 (PedsQL)}

The Pediatric Quality of Life Inventory 4.0 (PedsQL; Varni, Sied \& Kurtin, 1998) is considered to be the gold standard for measuring child health related QOL (Feeney, Desha, Ziviani $\&$ Nicholson, 2012). The PedsQL was designed to measure the core health dimensions specifically outlined by the WHO in 1948. The scale has been shown to accurately span ages of 2 to 18 years for both self-report and proxy-report while maintaining item and scale construct consistency (Varni, Seid \& Kurtin, 2001). Additionally, the PedsQL has been used to effectively assess QOL in children with various disabilities including autism spectrum disorder, IDD, spina bifida, heart disease and more (Kose et al., 2013; Murray et al., 2014; Uzark, Jones, Burwinkle \& Barni, 2003). Viecili and Weiss (2015) found that the core scales (physical, emotional, social, and school functioning) maintain their psychometric properties when measuring QOL for children with IDD. Multiple researchers have shown that scale scores can differentiate between children at risk for IDD and children with typical development (Basgul, Uneri \& Cakin-Memik, 2011; Lau, Chow $\&$ Lo, 2006). Given the consensus in the literature and strong psychometric properties, the PedsQL appears to be the best measure to assess health related QOL for children with IDD.

The PedsQL is a 23-item measure that assesses four core components: physical functioning (8 items), emotional functioning (5 items), social functioning (5 items), and school functioning (5 items). Researchers who have previously used the PedsQL with children with IDD have reported good internal consistency for the subscales $(\alpha=.78-.89)$ (Viecili \& Weiss, 2015).

The parent proxy-report form was used to assess parent's perception of their child's health related QOL. The instructions asked for parents to identify how much of a problem the various activities have been for their child over the previous one month. For example, physical functioning, "walking more than one block;" emotional functioning, "feeling afraid or scared;" social functioning, "getting along with other children;" school functioning, "paying attention in class." Answers were rated on a scale of 0 (never a problem) to 4 (almost always a problem). Items were reverse coded and transformed to a scale of 0 to $100(0=100,1=75,2=50,3=25,4=0)$. This transformation results in higher scores being translated into higher QOL. Subscale scores represent the mean of scale scores for the included items.

\section{Intervention}

Children participated in three separate 10week interventions $(1,2$, and 3$)$. The interventions followed the academic calendar, with intervention 1 being conducted in the winter semester (January to April), intervention 2 in the fall semester (September to December) and intervention 3 in the winter semester (January to April). Each intervention was identical and consisted of the same general format focused on improving FMS. An important feature of this design is that each participant acts as his/her own control when the intervention is removed.

Specific target skills were based on the Test of Gross Motor Development Version 3 (TGMD$3)$. The TGMD-3 includes six locomotor skills (i.e., running, skipping) and seven ball skills (i.e., overhand throw, two hand catch). Each of the 10 sessions focused on one locomotor skill and one ball skill, with all 13 skills being covered at least once during each intervention. The 10 weekly sessions occurred once a week for one hour at an urban midwestern university. 
The intervention was a free communitybased, evening program. Each session followed the same general routine, developed using best practices for children with IDD (Ketcheson et al. 2017). This routine consisted of 10-15 minutes of free play, 5-minute warm up, 35 minutes of direct instruction, and 5-minute cool down. Direct instruction activities included individual work with the coach, small group work, and full group activities. Data was collected at a separate session, one-week before the intervention began and oneweek after the intervention ended for all three interventions.

Each athlete was paired with a coach and worked in a 1:1 ratio throughout the session and maintained the pairing through the duration of each intervention. Coaches consisted of preservice physical education teachers or individuals in a closely related area of study (e.g., special education, exercise science). Coaches were trained through classroom pivotal response teaching (CPRT) skills, which are methods for behavioral intervention for children with autism spectrum disorder to be used in classroom settings (Stahmer, Suhrheinrich, Reed, Bolduc \& Schreibman, 2011). This framework has been previously used in gross motor intervention research for children with ASD (Ketcheson et al. 2017).

\section{Procedures}

Written informed consent was obtained from the child's parent before initial participation in the study. Following parent approval, an approved oral assent agreement was read to the child by the coaches to confirm their participation was voluntary. Parents completed a general demographic questionnaire and the PedsQL at the beginning and end of each FMS intervention. Over the year and a half of participation, a total of 30 intervention sessions and 6 data collection sessions occurred, for total of 36 sessions. A longitudinal design was employed examining participant's selfreport answers to the PedsQL.

\section{Statistical Procedures}

Means and standard deviations were calculated for each of the subscales at the six time points (i.e., pre and post each of the three interventions). Given the lack of power associated with only 10 participants for four dependent variables assessed six times statistical analyses were contradicted. Hence, we created line graphs that were generated in SPSS Version 25 to create a visual representation of the data. Means of the full sample $(\mathrm{N}=10)$ for the 4 subscales at each time point were graphed.

We conducted a visual analysis and modelled our research design after the single case experimental design for within groups (Lane \& Gast, 2013). Visual analysis of data displayed in graphs is a common method used in single case experimental design for ongoing evaluation of behavior change interventions (Lane \& Gast, 2013). Using visual analysis for this study allowed us to identify if there was a change in QOL for each of the three interventions and, importantly, if any gains noticed at the end of each intervention stabilized when the intervention was removed.

Additionally, visual analysis allowed us to compare the changes in QOL across all participants when each subscale was assessed individually. When there is visual evidence of change over time of individuals' response to the interventions, a clearer picture of the impact on the outcome variables are demonstrated (Crabtree, Ray, Schmidt, O’Connor \& Schmidt, 1989).

\section{RESULTS}

Participants attended most sessions as the average attendance for the 30 sessions (three interventions times 10 meetings per session) was 25 (SD=3.33). Internal consistency was determined using Cronbach's alpha for scores on each of the four subscales across the six time points. All of the subscales produced scores with good reliability (a score of .70 and above; Tavakol $\&$ Dennick, 2011) as follows: physical function ( $\alpha$ $=.82)$, emotional function $(\alpha=.85)$, social function $(\alpha=.85)$, and school function $(\alpha=.87)$.

The mean values of all 10 participants were computed and plotted for the four subscales at each of the six time points. A visual analysis shows that overall, there was a trend toward improved QOL for all subscales (see Figure 1). Our initial hypothesis, which stated an expectation of increased QOL during the interventions $(1,2$, and 3), was, in general, supported with a few exceptions. Also, we expected QOL to stay the same during the times between interventions (end of intervention 1 to the beginning of intervention 2 , end of intervention 2 to beginning of intervention 3). 
First, for emotional function, there was an increase during all three interventions. Additionally, there was a slight increase between the end of intervention 1 and the beginning of intervention 2 and a decrease between the end of intervention 2 and the beginning of intervention 3 (i.e., when the interventions were removed). Second, for social function, there was an increase during all three interventions. For social function, there was continued increase between the end of intervention 1 and the beginning of intervention 2 and a decrease between interventions 2 and 3 (i.e., when the interventions were removed). Third, for physical function, there was a decrease during the first intervention and increase during the second and third interventions. Between interventions, physical function increased between the end of first intervention and the beginning of the second intervention and stayed the same between the end of intervention 2 and beginning of intervention 3 . Lastly, for school function, there was no change during the first intervention or third intervention, but an increase during the second intervention. Additionally, there was no change between interventions 1 and 2 and 2 and 3 when the interventions were removed.

\section{Figure 1: QOL Subscales Across All Time Points}

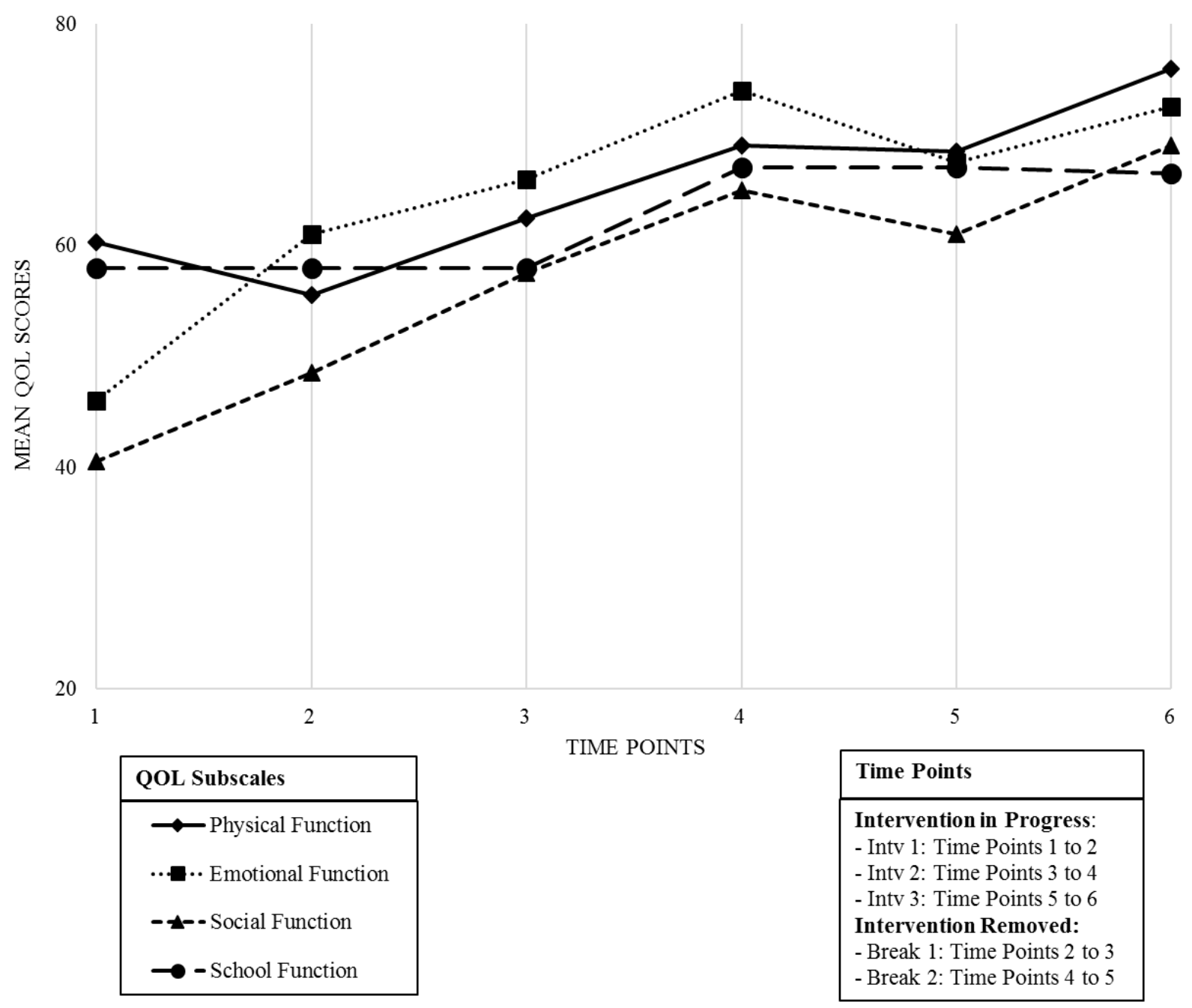

*Note: PedsQL Full Mean Range is 0 to 100 . 


\section{DISCUSSION}

The purpose of this study was to evaluate the longitudinal impact of a three part FMS intervention on enhancing QOL for children with IDD. In general, it appears that the intervention served as an effective tool for improving the multiple components of health related QOL for children with IDD. The sample trended toward increased QOL throughout the entire duration of the study. Both emotional and social functioning increased during each of the 3 interventions. Physical functioning increased during the second and third intervention but not the first intervention. School functioning increased during the second intervention only and not the first and third intervention.

The changes in QOL shown in this study seem to be most prominent in social and emotional functioning, compared to physical and school functioning. This finding was somewhat unexpected given that we anticipated physical functioning to be more directly impacted because the primary focus of the interventions was on the development of FMS. We next discuss each QOL dimension separately.

First, the social functioning area of QOL showed the largest changes in this study. The questions on this subscale of the PedsQL consists of getting along with other children, keeping up when playing with other children, being able to do the same things as children their age, and getting teased by other children. There are four potential reasons why the current intervention may have influenced social functioning QOL. First, although the intervention specifically focused on FMS improvement it was delivered in a group setting of 10 children where plenty of social interaction occurred before, during and after each meeting time. Second, each child was paired with a coach who provided lots of instruction, positive feedback, and sought to create a team-like atmosphere. Hence the interventions had strong social components to them making it plausible that the increased social interaction resulted in increased social QOL. Third, most of the children did not have access to sport activities with their peers on a regular basis or to work individually with a coach who is specifically trained to provide a positive psychosocial climate. Additionally, children with IDD often lack social and communication skills which are barriers to developing friendships inside and outside of school. As a result, building a relationship with the coach and engaging with other children with similar abilities may be particularly powerful influences on social QOL compared to neurotypically children. Finally, children with IDD are often at risk for being teased and ostracized during regular school life. As researchers we were particularly mindful that the meeting times would be devoid of any such negative behavior. Hence it is quite plausible that the creation of a psychosocial safe space during the time of the intervention also contributed to enhanced social QOL.

Second, emotional functioning QOL also increased during each of the three intervention programs. Given that social interactions play a large role in people's emotional lives it is plausible that the positive social interactions among peers and between participants and coaches also generated positive affective states (e.g., increased happiness) and reduced negative affective states (e.g., anxiety). We believe that the net effect of such socially induced emotions led to enhanced emotional QOL. Similar, to social QOL it is also plausible that the safe space created during the meeting times also contributed to enhanced emotional QOL.

Third, for physical QOL, there are many factors that could have played a role in the decrease during the first intervention and increases in the second and third intervention. We next focus on two potential reasons. First, many of the PedsQL physical functioning questions are directed toward activities of daily living, such as lifting something heavy, taking a bath or shower by themselves, or having aches. FMS development during the multiple interventions is likely to increase PA and increased PA is likely to lead to increased fitness and one's ability to engage in various daily activities. However, such gains may take time and as a result may have failed to show up during the first intervention period. Second, the FMS intervention specifically focused on the locomotor and ball skills and such skill enhancement may not lead directly to an initial (first intervention) increased ability to engage in activities of daily life, but contribute later on after a second and third round of FMS skill development. 
Lastly, for school functioning, the only change in QOL happened during the second intervention. We are unsure why parents noted an increase in school QOL for the second intervention but failed to recognize any improvement during the first and third intervention. It is possible that various factors outside of the control of children (e.g., course difficulty, subjects taken, teachers, etc.) differentially influenced this QOL dimension while any FMS or social/emotional QOL gains experienced had little influence on school QOL specific functioning.

Cumulatively, across the four QOL dimensions and three intervention periods increases were noted in nine of 12 instances. For the four QOL dimensions when the intervention was removed the first time three of four QOL dimensions continued to increase while one remained stable. During the time between the second and third interventions all four QOL dimensions remained stable or dropped. All four dimensions demonstrated higher QOL at the final data point compared to the initial baseline. In general, despite some fluctuations across the course of the study, the pattern of results was supportive as increases in the four QOL dimensions mostly increased during the interventions (9/12) and were stable or dropped when the intervention was removed (5/8). These preliminary results are encouraging and certainly warrant continued research with larger samples using a control group and examining the potential mechanisms behind QOL change.

\section{Limitations}

There are a few limitations to the current study. First, there was no control group included in the study although each participant served as their own control when they were not participating in the intervention. Second, outside factors were not measured to determine their external influence on the participants and their QOL. However, given the continuous trend of QOL improvement over the entire 1.5 years of the study, it is reasonable to think that participation in the various interventions had a positive and potentially cumulative impact on the participants' QOL. Third, the sample size was small. Though the sample was small, we were able to obtain complete data at six time points over 1.5 years for all participants. This is a large accomplishment given the accessibility of this particular population. Longitudinal studies are valuable as they provide a more representative picture of QOL compared to cross sectional research that assesses QOL at one moment in time. More research is needed to evaluate QOL overtime for children with IDD while also assessing more external factors, such as therapy programs or other extracurricular PA programs, that may positively impact these various areas of life functioning. Additionally, adding a waitlist control or use of a different PA (e.g., sport based) intervention program could be beneficial for direct comparison of program impact for participants.

\section{Conclusions}

Longitudinal research on how FMS interventions can enhance multi-dimensional QOL for young children with IDD is lacking and the current study addresses this research gap. Although preliminary, the current study provides initial support for the value of FMS interventions in enhancing QOL of life across physical, social and emotional functioning. As PA participation continues to decrease for children with IDD, while IDD diagnoses continue to increase, feasible programming can not only impact physical functioning, but also improve QOL, which is essential for overall health. Researchers should continue to examine how and why various areas of QOL can be improved through FMS interventions.

\section{REFERENCES}

American Psychiatric Association. (2013). Diagnostic and statistical manual of mental disorders (5th ed.). Arlington, VA: American Psychiatric Publishing.

Başgül, Ş. S., Üneri, Ö. Ş., \& Çakın-Memik, N. (2011). Parents' perception of the quality of life of children with intellectual disabilities. Turkish Journal of Pediatrics, 53, 541-546

Bishop, J. C., \& Pangelinan, M. (2018). Motor skills intervention research of children with disabilities. Research in Developmental Disabilities, 74, 14-30.

Bossink, L. W., van der Putten, A. A., \& Vlaskamp, C. (2017). Understanding low levels of physical activity in people with intellectual disabilities: A systematic review to identify barriers and facilitators. Research in Developmental Disabilities, 68, 95-110.

Boyle, C. A., Boulet, S., Schieve, L. A., Cohen, R. A., Blumberg, S. J., Yeargin-Allsopp, M., ... 
\& Kogan, M. D. (2011). Trends in the prevalence of developmental disabilities in US children, 1997-2008. Pediatrics, 127, 1034-1042.

Esposito, P. E., MacDonald, M., Hornyak, J. E., \& Ulrich, D. A. (2012). Physical activity patterns of youth with Down syndrome. Intellectual and Developmental Disabilities, 50, 109-119.

Feeney, R., Desha, L., Ziviani, J., \& Nicholson, J. M. (2012). Health-related quality-of-life of children with speech and language difficulties: A review of the literature. International Journal of Speech-Language Pathology, 14, 59-72.

Felce, D., \& Perry, J. (1995). Quality of life: Its definition and measurement. Research in Developmental Disabilities, 16, 51-74.

Gregor, S., Bruni, N., Grkinic, P., Schwartz, L., McDonald, A., Thille, P., ... \& Jachyra, P. (2018). Parents' perspectives of physical activity participation among Canadian adolescents with Autism Spectrum Disorder. Research in Autism Spectrum Disorders, 48, 53-62.

Guyatt, G. H., Feeny, D. H., \& Patrick, D. L. (1993). Measuring health-related quality of life. Annals of Internal Medicine, 118, 622629.

Johnson, C. C. (2009). The benefits of physical activity for youth with developmental disabilities: a systematic review. American Journal of Health Promotion, 23, 157-167.

Ketcheson, L., Hauck, J., \& Ulrich, D. (2017). The effects of an early motor skill intervention on motor skills, levels of physical activity, and socialization in young children with autism spectrum disorder: A pilot study. Autism, 21, 481-492.

Kose, S., Erermis, S., Ozturk, O., Ozbaran, B., Demiral, N., Bildik, T., \& Aydin, C. (2013). Health Related Quality of Life in children with Autism Spectrum Disorders: The clinical and demographic related factors in Turkey. Research in Autism Spectrum Disorders, 7, 213-220.

Lau, K. M., Chow, S. M., \& Lo, S. K. (2006). Parents' perception of the quality of life of preschool children at risk or having developmental disabilities. Quality of Life Research, 15, 1133-1141.
Memari, A. H., Panahi, N., Ranjbar, E., Moshayedi, P., Shafiei, M., Kordi, R., \& Ziaee, V. (2015). Children with autism spectrum disorder and patterns of participation in daily physical and play activities. Neurology Research International, 2015, 1-7.

Muldoon, M. F., Barger, S. D., Flory, J. D., \& Manuck, S. B. (1998). What are quality of life measurements measuring?. BMJ, 316, 542-545.

Murray, C. B., Holmbeck, G. N., Ros, A. M., Flores, D. M., Mir, S. A., \& Varni, J. W. (2014). A longitudinal examination of health-related quality of life in children and adolescents with spina bifida. Journal of Pediatric Psychology, 40, 419-430.

Penedo, F. J., \& Dahn, J. R. (2005). Exercise and well-being: a review of mental and physical health benefits associated with physical activity. Current Opinion in Psychiatry, 18, 189-193.

Pickard, A. S., \& Knight, S. J. (2005). Proxy evaluation of health-related quality of life: a conceptual framework for understanding multiple proxy perspectives. Medical Care, 43, 493-499.

Potvin, M. C., Snider, L., Prelock, P. A., WoodDauphinee, S., \& Kehayia, E. (2015). Health-related quality of life in children with high-functioning autism. Autism, 19, 14-19.

Robinson, L. E., Stodden, D. F., Barnett, L. M., Lopes, V. P., Logan, S. W., Rodrigues, L. P., \& D'Hondt, E. (2015). Motor competence and its effect on positive developmental trajectories of health. Sports Medicine, 45, 1273-1284.

Robinson, L. E., Stodden, D. F., Barnett, L. M., Lopes, V. P., Logan, S. W., Rodrigues, L. P., \& D'Hondt, E. (2015). Motor competence and its effect on positive developmental trajectories of health. Sports Medicine, 45, 1273-1284.

Stahmer, A., Suhrheinrich, J., Reed, S., Bolduc, C., \& Schreibman, L. (2011). Classroom pivotal response teaching: A guide to effective implementation. Guliford Press: New York, NY.

Strong, W. B., Malina, R. M., Blimkie, C. J., Daniels, S. R., Dishman, R. K., Gutin, B., ... \& Rowland, T. (2005). Evidence based 
physical activity for school-age youth. The Journal of Pediatrics, 146, 732-737.

Tavakol, M., \& Dennick, R. (2011). Making sense of Cronbach's alpha. International Journal of Medical Education, 2, 53-55.

Tyler, K., MacDonald, M., \& Menear, K. (2014). Physical activity and physical fitness of school-aged children and youth with autism spectrum disorders. Autism Research and Treatment, 2014. 1-6.

Upton, P., Lawford, J., \& Eiser, C. (2008). Parentchild agreement across child health-related quality of life instruments: a review of the literature. Quality of Life Research, 17, 895913.

US Department of Health and Human Services. (2018). Physical Activity Guidelines for Americans, 2nd edition. Washington, DC: US Department of Health and Human Services.

Uzark, K., Jones, K., Burwinkle, T. M., \& Varni, J. W. (2003). The Pediatric Quality of Life Inventory ${ }^{\mathrm{TM}}$ in children with heart disease. Progress in Pediatric Cardiology, 18, 141149.

Varni, J. W., Seid, M., \& Kurtin, P. S. (1998). Pediatric quality of life inventory version 4.0. Mapi Research Institute: Lyon, France.

Varni, J. W., Seid, M., \& Kurtin, P. S. (2001). PedsQL ${ }^{\text {TM }}$ 4.0: Reliability and validity of the Pediatric Quality of Life Inventory ${ }^{\mathrm{TM}}$ Version 4.0 Generic Core Scales in healthy and patient populations. Medical Care, 39, 800-812.

Viecili, M. A., \& Weiss, J. A. (2015). Reliability and validity of the Pediatric Quality of Life Inventory with individuals with intellectual and developmental disabilities. American Journal on Intellectual and Developmental Disabilities, 120, 289-301.

Wallander, J. L., Schmitt, M., \& Koot, H. M. (2001). Quality of life measurement in children and adolescents: issues, instruments, and applications. Journal of Clinical Psychology, 57, 571-585.

Warburton, D. E., Nicol, C. W., \& Bredin, S. S. (2006). Health benefits of physical activity: the evidence. Cmaj, 174(6), 801-809. WoodDauphinee, S. (1999). Assessing quality of life in clinical research: from where have we come and where are we going?. Journal of Clinical Epidemiology, 52, 355-363.
World Health Organization. (1948). Preamble to the Constitution of the World Health Organization as adopted by the International Health Conference, New York, 19-22 June, 1946; signed on 22 July 1946 by the representatives of 61 States (Official Records of the World Health Organization, no. 2, p. 100) and entered into force on 7 April 1948. http://www. who. int/governance/eb/who_constitution_en. pdf.

How to cite this article: Snapp, E.E., Ketcheson, L.R., Martin, J.J. and Centeio, E.E. (2020). Enhancing Quality of Life for Children with Intellectual and Developmental Disabilities. Int $\mathrm{J}$ Disabil Sports Health Sci;3(1):33-41. https://doi.org/10.33438/ijdshs.713634 